\title{
A temperature correction method for expanding atmospheres
}

\author{
W.-R. Hamann and G. Gräfener \\ Professur Astrophysik, Universität Potsdam, Am Neuen Palais 10, 14469 Potsdam, Germany \\ Received 2 June 2003 / Accepted 22 August 2003

\begin{abstract}
Model atmospheres form the basis for the interpretation of stellar spectra. A major problem in those model calculations is to establish the temperature stratification from the condition of radiative equilibrium. Dealing with non-LTE models for spherically expanding atmospheres of Wolf-Rayet stars, we developed a new temperature correction method. Its basic idea dates back to 1955 when it was proposed by Unsöld for grey, static and plane-parallel atmospheres in LTE. The equations were later generalized to the non-grey case by Lucy. In the present paper we furthermore drop the Eddington approximation, proceed to spherical geometry and allow for expansion of the atmosphere. Finally the concept of an "approximate lambda operator" is employed to speed up the convergence. Tests for Wolf-Rayet type models demonstrate that the method works fine even in situations of strong non-LTE.
\end{abstract}

Key words. stars: winds, outflows - stars: mass-loss - stars: Wolf-Rayet - radiative transfer - stars: atmospheres

\section{Introduction}

Model atmospheres form the basis for the interpretation of stellar spectra. Only by comparison with numerical simulations, the parameters and surface abundances of stars can be derived in quantitative spectral analyses. A major problem in those model calculations is to establish the temperature stratification of the stellar atmosphere in such a way that the law of energy conservation is fulfilled accurately. Although the concept is simple, the numerical implementation is not straightforward.

We have been confronted with that problem once more when developing improved non-LTE models for spherically expanding atmospheres of hot stars, especially Wolf-Rayet stars. After using different approaches in the past, we now developed the temperature correction method described in the present paper. The new method has been applied already for a large variety of models and proved to be stable, accurate and efficient. It is based on a method devised by Unsöld $(1951,1955)$ originally for grey, static and plane-parallel atmospheres in LTE. The equations were generalized to the non-grey case by Lucy (1964; see also Mihalas 1978). In the present paper we furthermore drop the Eddington approximation, proceed to spherical geometry and allow for expansion of the atmosphere. Finally the concept of an "approximate lambda operator" is employed to speed up the convergence. This sufficiently compensates for the systematic under-estimate of the temperature corrections due to the LTE assumption which is inherent to the method.

After the stage is set for atmospheres in radiative equilibrium in the following section, we will in Sect. 3 stepwise

Send offprint requests to: W.-R. Hamann, e-mail: wrh@astro.physik. uni-potsdam.de generalize Unsöld's method until it can be applied for spherically expanding non-LTE atmospheres. A few test results are shown in the final section (Sect. 4).

\section{Model atmospheres in radiative equilibrium}

A stellar atmosphere is termed to be in radiative equilibrium ( $\mathrm{RE}$ ), if radiation is the only mechanism of energy transfer. In this case the absorbed and the emitted radiation energy must be balanced in each volume element. Adopting Mihalas' (1978) definitions and notation we can write this as

$$
\begin{aligned}
\int_{\nu=0}^{\infty} \frac{1}{2} \int_{\mu=-1}^{1} \eta_{\nu}(\mu) \mathrm{d} \mu \mathrm{d} \nu= \\
\int_{\nu=0}^{\infty} \frac{1}{2} \int_{\mu=-1}^{1} \kappa_{v} I_{\nu}(\mu) \mathrm{d} \mu \mathrm{d} \nu .
\end{aligned}
$$

In moving media it is favorable to measure the frequencies in the Co-Moving Frame (CMF) of reference, in which the considered volume element is at rest. In that frame the absorption coefficient $\kappa_{v}$ is isotropic; when assuming complete angle redistribution, the emissivity $\eta_{v}$ is isotropic as well, and so is the source function $S_{v}=\eta_{v} / \kappa_{v}$. With the definition of the angleaveraged intensity

$J_{v}=\frac{1}{2} \int_{-1}^{1} I_{v}(\mu) \mathrm{d} \mu$

the equation of RE becomes

$\int_{v=0}^{\infty} \kappa_{v}\left(S_{v}-J_{v}\right) \mathrm{d} v=0$ 
Let us assume that $S_{v}$ is a known (monotonic) function of the temperature. This obviously holds for local thermodynamical equilibrium (LTE) where $S_{v}=B_{v}(T)$; the non-LTE case will be discussed below. Then, for a given radiation field $J_{v}$ and opacity $\kappa_{v}$ the local temperature $T$ can be obtained from Eq. (3).

However, in a model calculation the radiation field $J$ is not known a priori, but follows from radiative transfer and depends on the overall temperature field, not only at the considered spot. Symbolically the equation of radiative transfer might be considered as a linear mapping $\mathbf{S} \rightarrow \mathbf{J}$, represented by a linear operator $\Lambda$, i.e.

$$
\mathbf{J}=\boldsymbol{\Lambda} \mathbf{S}(n) \text {. }
$$

Here we have already indicated the more general case of non-LTE, where the source function $S_{v}$ depends only indirectly on the temperature, but can be expressed by the atomic level population numbers $n$.

These population numbers, on the other hand, must at each point fulfill the equations of statistical equilibrium. If we arrange the population numbers at the considered spatial point in a row vector $\boldsymbol{n}$, this system of equations which is linear in $\boldsymbol{n}$ can be written as

$$
\boldsymbol{n} \circ \mathbf{P}(\mathbf{J})=\boldsymbol{b}
$$

where the right-hand side vector $\boldsymbol{b}$ is essentially zero except for those elements which express number conservations. The coefficient matrix $\mathbf{P}$ describes the transition probability between the different atomic levels, containing radiative processes which depend on the radiation field, and collisional transitions depending on $T$.

The problem of non-LTE calculations is to solve consistently both sets of equations, the radiative transfer Eq. (4) and the statistical equations (Eq. (5)). The former couples in space, and the latter in frequency, so that the whole set of variables is coupled (cf. also Hamann 2003). Many recent non-LTE codes simply solve both sets of equations in turn, using the method of "approximate lambda operators" to achieve convergence (see Sect. 3.5).

The present paper now addresses the question, how to calculate a consistent temperature stratification from the Radiative Equilibrium condition when solving the non-LTE problem. One possible strategy, which we applied in earlier versions of our model code (Hamann \& Wessolowski 1990), is to add $\mathrm{RE}$ as an additional equation to the system of statistical equations (Eq. (5)), introducing $T$ as a further independent variable in addition to the population numbers $n$. This method did not work stable with our recent, metal line blanketed version of our code.

An alternative and more successful approach is described in the present paper. Being a temperature correction scheme, the temperature is updated within the iteration cycle between radiation transfer and statistical equations by means of a separate calculation. Clearly one would not expect fast convergence of such a method, because the RE equation is not solved together with the other constraint equations and consistence is only obtained iteratively. But the advantage of such a temperature correction method is its straightforward formulation and implementation, which allows to trace the effect of each term and assumption in a direct way.

An iterative method must achieve a sufficient rate of convergence in order to be useful. Consider the most simple temperature correction method, historically termed "lambda iteration". A given source function $S_{v}$ and radiation field $J_{v}$ may in general not satisfy RE, i.e. the right-hand side of Eq. (3) is not zero but

$\int_{v=0}^{\infty} \kappa_{v}\left(S_{v}-J_{v}\right) \mathrm{d} v=\Delta E$.

Now we are searching for a correction of the source function, $\Delta S_{v}$, such that

$\int_{v=0}^{\infty} \kappa_{v}\left(S_{v}+\Delta S_{v}-J_{v}\right) \mathrm{d} v=0$

From comparing the last two equations, we obtain for this $\Delta S_{v}$

$\int_{v=0}^{\infty} \kappa_{v} \Delta S_{v} \mathrm{~d} v=\Delta E$.

Let us assume for a moment that the opacity is "grey", and the source function is the Planck function (LTE). Then, $\Delta B=$ $\Delta E / \kappa$ where the (bolometric) Planck function can be expanded for small temperature corrections as $\Delta B \approx \frac{4 \sigma}{\pi} T^{3} \Delta T$. Thus the temperature correction is readily obtained as

$\Delta T=\frac{\pi}{4 \sigma T^{3}} \frac{\Delta E}{\kappa}$.

This straightforward approach does not converge with a reasonable rate, as soon as parts of the spectrum are optically thick. The problem is due to the fact that the effect of a correction in one point only propagates by one optical pathlength per iteration.

\section{Unsöld's iteration method}

\subsection{The grey, plane-parallel, static case}

In order to overcome the convergence difficulties just mentioned, Unsöld (1951, 1955) proposed a method which he called Strom-Iteration. The present paper relies on the same concept. Its basic idea is to combine the RE equation with the equation of radiative transfer. For a plane-parallel and static medium, the latter is written as

$-\mu \frac{\mathrm{d} I_{v}}{\mathrm{~d} t}=\kappa_{v}\left(S_{v}-I_{v}\right)$

where $t$ is the geometrical depth coordinate with $t=0$ at the stellar surface. Integration over $\mathrm{d} \mu$ and $\mu \mathrm{d} \mu$ gives the 0th Moment Equation (0. MEQ)

$-\frac{\mathrm{d} H_{v}}{\mathrm{~d} t}=\kappa_{v}\left(S_{v}-J_{v}\right)$

and the 1st Moment Equation (1.MEQ)

$\frac{\mathrm{d} K_{v}}{\mathrm{~d} t}=\kappa_{v} H_{v}$ 
with the moments of the intensity $I_{v}$ being defined as

$$
\begin{aligned}
& J_{v}=\frac{1}{2} \int_{-1}^{1} I_{v}(\mu) \mathrm{d} \mu \quad H_{v}=\frac{1}{2} \int_{-1}^{1} I_{v}(\mu) \mu \mathrm{d} \mu \\
& K_{v}=\frac{1}{2} \int_{-1}^{1} I_{v}(\mu) \mu^{2} \mathrm{~d} \mu .
\end{aligned}
$$

Frequency integral of the 0 . MEQ gives

$\int_{0}^{\infty} \frac{\mathrm{d} H_{v}}{\mathrm{~d} t} \mathrm{~d} v=\int_{0}^{\infty} \kappa_{v}\left(S_{v}-J_{v}\right) \mathrm{d} v$

where the right-hand side is zero in Radiative Equilibrium. Therefore the frequency-integrated (bolometric) Eddington Flux

$H=\int_{0}^{\infty} H_{v} \mathrm{~d} v$

is constant and given by the stellar effective temperature

$H(t) \equiv H_{0}=\frac{\sigma}{4 \pi} T_{\mathrm{eff}}^{4}$,

the well-known equivalence of RE and flux conservation.

Introducing now Unsöld's method first in its original form, we consider a frequency-independent (grey) opacity and define some further frequency-integrated quantities

$J=\int_{0}^{\infty} J_{v} \mathrm{~d} v \quad K=\int_{0}^{\infty} K_{v} \mathrm{~d} v \quad S=\int_{0}^{\infty} S_{v} \mathrm{~d} v$.

With these definitions, the two Moment Equations (Eqs. (11) and (12)) can be integrated over frequency and yield

(0. MEQ) $-\frac{\mathrm{d} H}{\mathrm{~d} t}=\kappa(S-J)$

and

(1. MEQ) $\frac{\mathrm{d} K}{\mathrm{~d} t}=\kappa H$.

The 1. MEQ can be integrated over the optical depth $\tau$, defined as $\mathrm{d} \tau=\kappa \mathrm{d} t$, giving

$K(\tau)=\int_{\tau^{\prime}=0}^{\tau} H\left(\tau^{\prime}\right) \mathrm{d} \tau^{\prime}+$ const.

While Unsöld assumed the Eddington (diffusion) approximation $\frac{K}{J}=\frac{1}{3}$, we introduce "variable Eddington factors" $f=\frac{K}{J}$ and $h=\frac{H}{J}$ here. In the numerical calculations, these Eddington factors are obtained from solving the angle-dependent (ray-byray) radiation transport equation, and are updated from time to time in the overall iteration scheme. Fixing also the integration constant at the boundary value, $J(\tau)$ is finally expressed as

$J(\tau)=\frac{1}{f(\tau)} \int_{\tau^{\prime}=0}^{\tau} H\left(\tau^{\prime}\right) \mathrm{d} \tau+\frac{H(0)}{h(0)}$.

Now consider the integral of radiative equilibrium (cf. Eq. (6)), in the grey case simply

$\kappa(S-J)=\Delta E$ which does not vanish for any given $S$ and $J$. In this equation, $J$ can be expressed by means of Eq. (21):

$\frac{\Delta E(\tau)}{\kappa(\tau)}=S(\tau)-\frac{1}{f(\tau)} \int_{\tau^{\prime}=0}^{\tau} H\left(\tau^{\prime}\right) \mathrm{d} \tau^{\prime}-\frac{H(0)}{h(0)}$

for any given source function $S(\tau)$ which might not satisfy RE. Now a correction $\Delta S$ is added to the source function, such that $\mathrm{RE}$ is retained (i.e. $\Delta E(\tau) \equiv 0$ ), and flux is conserved (i.e. $\left.H(\tau) \equiv H_{0}\right)$ at all depths:

$0 \quad=S(\tau)+\Delta S-\frac{1}{f(\tau)} \int_{\tau^{\prime}=0}^{\tau} H_{0} \mathrm{~d} \tau^{\prime}-\frac{H_{0}}{h(0)}$.

The difference of the last two equations yields the required correction for the source function,

$$
\begin{aligned}
\Delta S(\tau)= & -\frac{\Delta E(\tau)}{\kappa(\tau)} \\
& +\frac{1}{f(\tau)} \int_{\tau^{\prime}=0}^{\tau}\left(H_{0}-H\left(\tau^{\prime}\right)\right) \mathrm{d} \tau^{\prime}+\frac{H_{0}-H(0)}{h(0)} .
\end{aligned}
$$

Assuming that $\Delta S$ scales with temperature in the same way as in LTE, the temperature correction finally becomes

$\Delta T(\tau)=\frac{\pi}{4 \sigma[T(\tau)]^{3}} \Delta S(\tau)$

\subsection{The non-grey generalization}

The temperature correction method devised by Unsöld, as described in the preceding subsection, was generalized to the nongrey case by Lucy (1964; see also Mihalas 1978) to what became known as the "Unsöld-Lucy method". The basic idea is to introduce suitably weighted means of the (now non-grey) opacity, such that the integrals over products (like $\kappa_{v} J_{v}$ ) can still be replaced by products of (now frequency-integrated) quantities, similar to the grey case. The appropriate definitions are

$\kappa_{S}=\frac{\int_{0}^{\infty} \kappa_{v} S_{v} \mathrm{~d} v}{S}$,

$\kappa_{J}=\frac{\int_{0}^{\infty} \kappa_{v} J_{v} \mathrm{~d} v}{J}$

and

$\kappa_{H}=\frac{\int_{0}^{\infty} \kappa_{v} H_{v} \mathrm{~d} v}{H}$

In order to keep the Eddington factors $f$ and $h$ variable, we furthermore define intensity-weighted means of $f_{v}$ and $h_{v}$,

$f_{J}=\frac{\int_{0}^{\infty} f_{v} J_{v} \mathrm{~d} v}{J}$

and

$h_{J}=\frac{\int_{0}^{\infty} h_{v} J_{v} \mathrm{~d} v}{J}$.

The dependence of variables on the depth $(\tau$ or $t)$ is only explicitely written when not being obvious. With the help of 
these means, the frequency-integrated Moment Equations look similar to the grey case (cf. Eqs. (18) and (19)):

$$
\begin{aligned}
& \text { (0. MEQ) }-\frac{\mathrm{d} H}{\mathrm{~d} t}=\kappa_{S} S-\kappa_{J} J \\
& \text { (1. MEQ) } \frac{\mathrm{d}\left(f_{J} J\right)}{\mathrm{d} t}=\kappa_{H} H .
\end{aligned}
$$

Proceeding in analogy to the grey case, the 1. MEQ is integrated over depth,

$f_{J}(t) J(t)=\int_{t^{\prime}=0}^{t} \kappa_{H}\left(t^{\prime}\right) H\left(t^{\prime}\right) \mathrm{d} t^{\prime}+$ const.

where the integration constant is again fixed by the boundary value, thus yielding

$J(t)=\frac{1}{f_{J}(t)} \int_{t^{\prime}=0}^{t} \kappa_{H}\left(t^{\prime}\right) H\left(t^{\prime}\right) \mathrm{d} t^{\prime}+\frac{H(0)}{h_{J}(0)}$.

Now this expression for $J$ is inserted into the RE integral,

$\kappa_{S} S-\kappa_{J} J=\Delta E$,

resulting in

$$
\begin{aligned}
\frac{\Delta E(t)}{\kappa_{S}(t)}= & S(t) \\
& -\frac{\kappa_{J}(t)}{\kappa_{S}(t)}\left[\frac{1}{f_{J}(t)} \int_{t^{\prime}=0}^{t} \kappa_{H}\left(t^{\prime}\right) H\left(t^{\prime}\right) \mathrm{d} t^{\prime}+\frac{H(0)}{h_{J}(0)}\right]
\end{aligned}
$$

for any given source function $S$ not yet satisfying the RE condition. If now a suitable correction $\Delta S$ is added, RE and flux conservation are retained:

$$
\begin{aligned}
0= & S(t)+\Delta S(t) \\
& -\frac{\kappa_{J}(t)}{\kappa_{S}(t)}\left[\frac{1}{f_{J}(t)} \int_{t^{\prime}=0}^{t} \kappa_{H}\left(t^{\prime}\right) H_{0} \mathrm{~d} t^{\prime}-\frac{H_{0}}{h_{J}(0)}\right] .
\end{aligned}
$$

The difference of the last two equations yields for $\Delta S$

$$
\begin{aligned}
\Delta S(t)= & -\frac{\Delta E(t)}{\kappa_{S}(t)} \\
& +\frac{\kappa_{J}(t)}{\kappa_{S}(t)} \frac{1}{f_{J}(t)} \int_{t^{\prime}=0}^{t} \kappa_{H}\left(t^{\prime}\right)\left(H_{0}-H\left(t^{\prime}\right)\right) \mathrm{d} t \\
& +\frac{\kappa_{J}(t)}{\kappa_{S}(t)} \frac{H_{0}-H(0)}{h_{J}(0)},
\end{aligned}
$$

from which the temperature correction can be obtained finally via Eq. (26).

\subsection{The spherical case}

Remember (or see Mihalas 1978) that in polar coordinates $(r, \mu)$ with $\mu=\cos \theta$ the equation of radiation transfer becomes a partial differential equation,

$\mu \frac{\partial I}{\partial r}+\frac{1-\mu^{2}}{r} \frac{\partial I}{\partial \mu}=\kappa_{v}\left(S_{v}-I_{v}\right)$

because the angle between a specific ray and the radial direction changes along the ray. Integrating this equation over $\mathrm{d} \mu$ and $\mu \mathrm{d} \mu$ gives the 0 th Moment Equation

$\frac{\mathrm{d} \tilde{H}_{v}}{\mathrm{~d} r}=\kappa_{v}\left(\tilde{S}_{v}-\tilde{J}_{v}\right)$ and the 1st Moment Equation

$\frac{\mathrm{d}\left(q \tilde{K}_{v}\right)}{\mathrm{d} r}=-q_{v} \kappa_{v} \tilde{H}_{v}$

where the tilde denotes the incorporation of an $r^{2}$ factor, $\tilde{J}_{v}=$ $r^{2} J_{v}$ etc., and $q$ is the integrating ("sphericality") factor

$q(r)=r^{-2} \exp \left[\int_{1}^{r} \frac{3 f\left(r^{\prime}\right)-1}{r^{\prime} f\left(r^{\prime}\right)} \mathrm{d} r^{\prime}\right]$.

In order to proceed in analogy to the plane-parallel case, we define two further weighted means,

$(q f)_{J}=\frac{\int_{0}^{\infty} q_{v} f_{v} J_{v} \mathrm{~d} v}{J}$ and

$(q \kappa)_{H}=\frac{\int_{0}^{\infty} q_{v} \kappa_{v} H_{v} \mathrm{~d} v}{H}$

With their help, we can integrate the Moment Equations over frequency and obtain

(0. MEQ) $\frac{\mathrm{d} \tilde{H}}{\mathrm{~d} r}=\kappa_{S} \tilde{S}-\kappa_{J} \tilde{J}$

(1. MEQ) $\frac{\mathrm{d}\left((q f)_{J} \tilde{J}\right)}{\mathrm{d} r}=-(q \kappa)_{H} \tilde{H}$.

The latter equation can be integrated over radius $r$,

$(q f)_{J}(r) \tilde{J}(r)=\int_{r^{\prime}=r}^{R}(q \kappa)_{H}\left(r^{\prime}\right) \tilde{H}\left(r^{\prime}\right) \mathrm{d} r^{\prime}+$ const.

where the integration constant is fixed by the boundary values at $r=R$, giving for $\tilde{J}$

$$
\begin{aligned}
\tilde{J}(r)= & \frac{1}{(q f)_{J}(r)} \int_{r^{\prime}=r}^{R}(q \kappa)_{H}\left(r^{\prime}\right) \tilde{H}\left(r^{\prime}\right) \mathrm{d} r^{\prime} \\
& +\frac{(q f)_{J}(R)}{(q f)_{J}(r)} \frac{\tilde{H}(R)}{h_{J}(R)} .
\end{aligned}
$$

Now this expression for $\tilde{J}$ is inserted into the $r^{2}$-multiplied RE integral,

$\kappa_{S} \tilde{S}-\kappa_{J} \tilde{J}=\Delta \tilde{E}$,

and we obtain

$$
\begin{aligned}
\frac{\Delta \tilde{E}(r)}{\kappa_{S}(r)}= & \tilde{S}(r) \\
& -\frac{\kappa_{J}(r)}{\kappa_{S}(r)}\left[\frac{1}{(q f)_{J}(r)} \int_{r^{\prime}=r}^{R}(q \kappa)_{H}\left(r^{\prime}\right) \tilde{H}\left(r^{\prime}\right) \mathrm{d} r^{\prime}\right] \\
& -\frac{\kappa_{J}(r)}{\kappa_{S}(r)}\left[\frac{(q f)_{J}(R)}{(q f)_{J}(r)} \frac{\tilde{H}(R)}{h_{J}(R)}\right]
\end{aligned}
$$

for any given "wrong" $\tilde{S}(r)$. Again we rewrite this equation after adding a correction $\Delta \tilde{S}$ to the source function, such that Radiative Equilibrium and flux conservation are retained,

$0=\tilde{S}(r)+\Delta \tilde{S}(r)$

$$
\begin{aligned}
& -\frac{\kappa_{J}(r)}{\kappa_{S}(r)}\left[\frac{1}{(q f)_{J}(r)} \int_{r^{\prime}=r}^{R}(q \kappa)_{H}\left(r^{\prime}\right) \tilde{H}_{0} \mathrm{~d} r^{\prime}\right] \\
& -\frac{\kappa_{J}(r)}{\kappa_{S}(r)}\left[\frac{(q f)_{J}(R)}{(q f)_{J}(r)} \frac{\tilde{H}_{0}}{h_{J}(R)}\right] .
\end{aligned}
$$




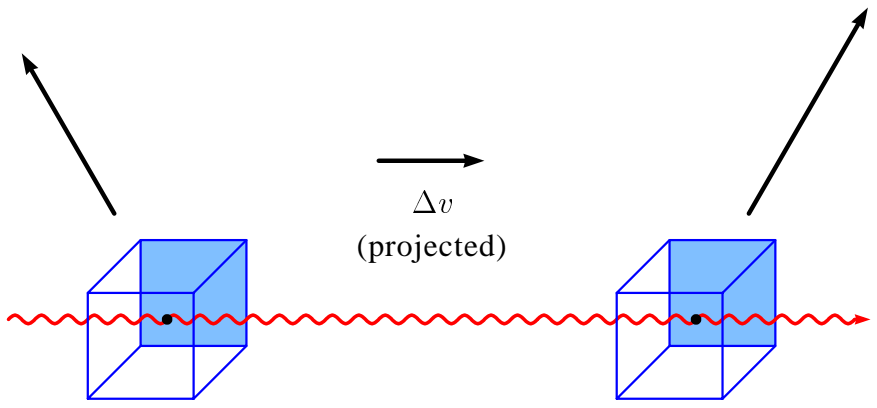

Fig. 1. In an expanding atmosphere, any two volume elements are differentially moving. Traveling photons appear Doppler-shifted, when their frequency is measured in different frames of reference comoving with the matter. The frequency shift between two points is proportional to their relative velocity, projected onto the photon ray.

The difference between the last two equations yields $\Delta \tilde{S}$,

$$
\begin{aligned}
\Delta \tilde{S}(r)= & -\frac{\Delta \tilde{E}(r)}{\kappa_{S}(r)} \\
& +\frac{\kappa_{J}(r)}{\kappa_{S}(r)} \frac{1}{(q f)_{J}(r)} \int_{r^{\prime}=r}^{R}(q \kappa)_{H}\left(r^{\prime}\right)\left(\tilde{H}_{0}-\tilde{H}\left(r^{\prime}\right)\right) \mathrm{d} r^{\prime} \\
& +\frac{\kappa_{J}(r)}{\kappa_{S}(r)} \frac{(q f)_{J}(R)}{(q f)_{J}(r)} \frac{\tilde{H}_{0}-\tilde{H}(R)}{h_{J}(R)}
\end{aligned}
$$

from which the temperature correction can be obtained in LTE analogy by

$\Delta T(r)=\frac{\pi}{4 \sigma[T(r)]^{3} r^{2}} \Delta \tilde{S}(r)$

\subsection{Generalization to expanding atmospheres}

The final step to be done is to generalize the method to the case of expanding atmospheres. The matter moves radially outward with a velocity $v(r)$, which is assumed to be monotonically growing. Therefore any two parcels of matter are receding from each other. The radiation transfer is formulated in the Co-Moving Frame (CMF), i.e. frequencies and radiation-field quantities are measured at each spatial point in the local rest frame of the moving matter. Due to the differential motion, a photon experiences a red-shift while traveling (cf. Fig. 1). Consequently, the CMF equation of radiation transport along a ray becomes a partial differential equation for the intensity $I_{v}$ :

$\pm \frac{\partial I_{v}^{ \pm}}{\partial z}-P \frac{v}{c} \frac{\partial I_{v}^{ \pm}}{\partial v}=\kappa_{v}\left(S_{v}-I_{v}\right)$.

Here $z$ is the spatial coordinate along the considered ray, and $P$ is the velocity gradient projected onto that ray,

$P=\mu^{2}\left(\frac{\mathrm{d} v}{\mathrm{~d} r}-\frac{v}{r}\right)+\frac{v}{r}=\mu^{2} w+\frac{v}{r}$

with the abbreviation $w=\frac{\mathrm{d} v}{\mathrm{~d} r}-\frac{v}{r}$ for the difference between the radial and the transversal velocity gradient.

Integrating the "ray-by-ray" CMF transfer Eq. (56) over $\mathrm{d} \mu$ and $\mu \mathrm{d} \mu$ gives the 0 . Moment Equation

$\frac{\partial \tilde{H}_{v}}{\partial r}-w \frac{v}{c} \frac{\partial \tilde{K}_{v}}{\partial x}-\frac{v}{r} \frac{v}{c} \frac{\partial \tilde{J}_{v}}{\partial v}=\kappa_{v}\left(\tilde{S}_{v}-\tilde{J}_{v}\right)$ and the 1. Moment Equation

$\frac{\partial\left(q_{v} \tilde{K}_{v}\right)}{-q_{v} \partial r}+w \frac{v}{c} \frac{\partial \tilde{N}_{v}}{\partial v}+\frac{v}{r} \frac{v}{c} \frac{\partial \tilde{H}_{v}}{\partial v}=\kappa_{v} \tilde{H}_{v}$

In addition to the moments of the radiation intensity defined earlier (Eq. (13)), a further (third) moment $\tilde{N}$ occurs here,

$\tilde{N}_{v}=r^{2} \frac{1}{2} \int_{-1}^{1} I_{\nu}(\mu) \mu^{3} \mathrm{~d} \mu$.

By applying the same weighted means as in the previous subsections, we can integrate the 0. Moment Equations over frequency and obtain

(0. MEQ) $\frac{\mathrm{d} \tilde{H}}{\mathrm{~d} r}+\frac{1}{c}\left[w \tilde{K}+\frac{v}{r} \tilde{J}\right]=\kappa_{S} \tilde{S}-\kappa_{J} \tilde{J}$.

This equation shows, that for an expanding atmosphere in radiative equilibrium, i.e. when the right-hand side vanishes, the CMF flux $\tilde{H}$ is not conserved. Instead, the photon field looses inevitably energy by doing mechanical work. Integration of Eq. (61) over radius yields

$$
\begin{aligned}
\tilde{H}_{0}(r)= & \tilde{H}_{0}(1) \\
& -\frac{1}{c} \int_{r^{\prime}=1}^{r}\left[w\left(r^{\prime}\right) \tilde{K}\left(r^{\prime}\right)+\frac{v\left(r^{\prime}\right)}{r^{\prime}} \tilde{J}\left(r^{\prime}\right)\right] \mathrm{d} r^{\prime}
\end{aligned}
$$

where we assumed that the flux at the inner boundary of the atmosphere, i.e. at $r=1$, is specified. Therefore, in contrast to the static case we have to calculate $\tilde{H}_{0}(r)$ and use this radiusdependent value for the further calculation.

This is in fact the only modification we make in order to account for the expansion, compared to the static case described in the preceding subsection. The frequency integral of the 1. Moment Equation Eq. (59) also yields additional "expansion terms" with frequency derivatives, in addition to the static case Eq. (47). However, in the next step this equation will be written twice, first for the "wrong" source function and a second time for the corrected $S$, and then the correction will be obtained from subtracting both equations. If we neglect the (very small) reaction of the expansion terms on the correction of the source function, these terms cancel out. Note that this approximation only affects the temperature correction, but not the converged solution of the method.

Thus, with the only modification of the radius-dependence of the pre-specified flux $\tilde{H}_{0}(r)$, the steps following Eq. (47) remain the same for the expanding case. The source function correction is obtained as

$$
\begin{aligned}
\Delta \tilde{S}(r)= & -\frac{\Delta \tilde{E}(r)}{\kappa_{S}(r)} \\
& +\frac{\kappa_{J}(r)}{\kappa_{S}(r)} \frac{1}{(q f)_{J}(r)} \int_{r^{\prime}=r}^{R}(q \kappa)_{H}\left(r^{\prime}\right)\left(\tilde{H}_{0}\left(r^{\prime}\right)-\tilde{H}\left(r^{\prime}\right)\right) \mathrm{d} r^{\prime} \\
& +\frac{\kappa_{J}(r)}{\kappa_{S}(r)} \frac{(q f)_{J}(R)}{(q f)_{J}(r)} \frac{\tilde{H}_{0}(R)-\tilde{H}(R)}{h_{J}(R)}
\end{aligned}
$$

from which the temperature correction can be obtained in LTE analogy (Eq. (55)). 


\subsection{Combination with the ALI method}

The correction for the source function, as provided by Unsöld's method, is composed of three terms (cf. Eq. (64)): the last (third) term evaluates the difference between the correct flux $\tilde{H}_{0}(R)$ and the actual value $\tilde{H}(R)$, both at the outer boundary $r=R$, and communicates this correction immediately to the inner points $r$ of the atmosphere.

The second (middle) term is also driven by the "flux error", integrating this difference from the boundary to the considered point $r$. Because the flux-weighted mean opacity appears under this integral, significant contributions are collected from optically thick parts of the atmosphere only. This information is immediately communicated to the point $r$.

The first term is just as obtained from direct application of the Radiative Equilibrium condition alone (cf. Eq. (8)). This term bears the whole burden to correct $T(r)$ in those outer parts of the atmosphere which are optically thin in terms of the fluxweighted mean opacity.

At first glance, this duty seems not to be a problem for the first term, as the "lambda iteration" works fine in optically thin situations where the feedback of the temperature on the local radiation intensity is small. However, in a realistic stellar atmosphere we encounter extremely optically-thick (e.g. resonance) lines in layers which are optically thin in the continuum and, therefore, in the flux-weighted mean opacity. Due to the high opacity peaks, the radiative equilibrium integral (cf. Eq. (3)) is completely dominated by those few line frequencies. The strong local feedback of a temperature correction to the local radiation field is only slowly communicated to distant points via the radiation transfer, i.e. the convergence problem of the classical "lambda iteration" is still not overcome here.

The same iteration problem is generally encountered in non-LTE modeling, when the radiative transfer equation (Eq. (4)) and constraint equations (Eq.(5)) are solved in turn. The powerful method developed in the 1980s to overcome these difficulties is termed "iteration with Approximate Lambda Operators" (ALO method, see, e.g., Hamann 1985, $1986,2003)$. Let us repeat briefly its basic idea. When the radiative transfer (Eq. (4)) is calculated from a given stratification of population numbers and temperature, this is called a "formal solution" yielding the radiation field $\mathbf{J}^{\mathrm{fs}}$. In a simple lambda iteration, this radiation field would enter the subsequent solution of the statistical equations (Eq. (5)). In the ALO method, however, an approximate radiation intensity $\mathbf{J}^{*}$ is used instead, which formally accounts in advance for the corrected source function which is just to be calculated from the constraint equations. The same idea can also be applied for the radiative equilibrium equation (see also Hauschildt 1992).

The most simple formulation is obtained when the "Approximate Lambda Operator" $\Lambda_{v}^{*}$ only accounts for the $l o$ cal feedback of the source function, which is a not too bad assumption in the optically thick case which is just the critical one we have to defeat. At a given radius and frequency, application of such a "diagonal" ALO just means multiplication with a number, and the approximate mean intensity becomes

$J_{v}^{*}=J_{v}^{\mathrm{fs}}+\Lambda_{v}^{*} \Delta S_{v}$
The application of this ALO scheme to the RE equation, and thus to the first term of the Unsöld-Lucy temperature correction, is straightforward. First, the "error" in the RE integral is calculated from the radiation field as obtained by the formal solution of the radiation transfer, i.e.

$\Delta E=\int_{v=0}^{\infty} \kappa_{v}\left(S_{v}-J_{v}^{\mathrm{fs}}\right) \mathrm{d} v=\kappa_{S} S-\kappa_{J} J^{\mathrm{fs}}$.

Next we re-write this equation with the source function corrected by $\Delta S$ such that RE is retained, but at the same instance using $J_{v}^{*}$ instead of $J_{v}^{\mathrm{fs}}$ in order to account for the feedback,

$$
\begin{aligned}
0 & =\int \kappa_{v}\left(S_{v}+\Delta S_{v}-J_{v}^{*}\right) \mathrm{d} v \\
& =\Delta E+\int \kappa_{v} \Delta S_{v} \mathrm{~d} v-\int \kappa_{v} \Lambda_{v}^{*} \Delta S_{v} \mathrm{~d} v
\end{aligned}
$$

where we have already inserted the definition of $J_{v}^{*}$ from Eq. (64) in the second line. For resolving this equation for $\Delta S$ we obviously need to define further opacity means, weighted with the (still unknown) $\Delta S_{v}$. Here we now introduce the plausible approximation that weighting with $S_{v}$, i.e. with the source function itself instead of its correction, will yield very similar results. Thus we apply $\kappa_{S}$ in the preceding equation, and define additionally the weighted mean

$(\kappa \Lambda)_{S}=\frac{\int \kappa_{v} \Lambda_{v}^{*} S_{v} \mathrm{~d} v}{S}$.

With its help, we obtain

$0=\Delta E+\kappa_{S} \Delta S-(\kappa \Lambda)_{S} \Delta S$,

and thus the "amplified" correction (now denoted with a superscript "A") becomes

$\Delta S^{\mathrm{A}}=-\frac{\Delta E}{\kappa_{S}-(\kappa \Lambda)_{S}}$.

Compared to the correction without using the ALO,

$\Delta S=-\frac{\Delta E}{\kappa_{S}}$,

an amplification by a factor

$A=\frac{\kappa_{S}}{\kappa_{S}-(\kappa \Lambda)_{S}}$

has been achieved, which amounts to about $10^{4}$ in outer parts of a Wolf-Rayet atmosphere, typically.

The specific choice of the (diagonal) Approximate Lambda Operator $\Lambda_{v}^{*}$ is not critical. Basically, it is sufficient to choose 0 for optically thin and 1 for optically thick frequencies. As a more elaborated guess, we estimate the photon escape probability to be $\mathrm{e}^{-\tau_{v}}$ from the optical depth $\tau_{v}$ to the nearest boundary. We write

$\Lambda_{v}^{*}=1-\frac{1-\mathrm{e}^{-\tau_{v} / \gamma}}{\tau / \gamma}$

with some adjustable $\gamma$ to allow for manual damping of the corrections which can be chosen as unity in most cases. 
For a simple estimate of the optical depth $\tau_{\nu}(r)$ we neglect here the expansion and assume $\kappa_{v}$ to scale with $r^{-2}$ in order to obtain the local expression

$$
\begin{aligned}
\tau_{v}(r) & =\min \left[\kappa_{v}(r) \int_{r}^{R} \frac{r^{2}}{r^{\prime 2}} \mathrm{~d} r^{\prime}, \kappa_{v}(r) \int_{1}^{r} \frac{r^{2}}{r^{\prime 2}} \mathrm{~d} r^{\prime}\right] \\
& =\kappa_{v}(r) \min \left[r\left(1-\frac{r}{R}\right), \quad r(r-1)\right] .
\end{aligned}
$$

A few more tricks were found to be useful in order to make the method more robust in practical applications.

- In the optically thick part of the atmosphere, the ALO amplification interferes badly with the second and third "flux correction" terms of the Unsöld-Lucy method which already do a good job there. Therefore we damp the ALO amplification in regions of high mean (e.g., Rosseland) optical depth $\tau_{\text {Ross }}$, by replacing the amplification factor $A$ by some $A^{\prime}$ defined as

$$
A^{\prime}=1+(A-1) \mathrm{e}^{-\tau_{\text {Ross }}} \text {. }
$$

- One might consider to amplify the temperature corrections as obtained in LTE analogy, the more the non-LTE source function deviates from the Planck function, by arguing that the former is less sensitive to the local temperature. However, this does not work well in practical tests. On the contrary, it turns out that smoother convergence is obtained when the temperature corrections are damped with increasing mean departure coefficient $S / B$. Therefore we replace $A^{\prime}$ by

$$
A^{\prime \prime}=1+\left(A^{\prime}-1\right) \min \left(\frac{S}{B}, \frac{B}{S}\right) \text {. }
$$

- It is favorable to calculate separate temperature corrections $\Delta T_{\mathrm{I}}, \Delta T_{\mathrm{II}}$ and $\Delta T_{\mathrm{III}}$ from each of the three terms in Eq. (64). Then we can define individual damping factors $d_{\mathrm{I}}$, $d_{\text {II }}$ and $d_{\text {III }}$ when composing the total temperature correction $\Delta T$,

$$
\Delta T=d_{\mathrm{I}} \Delta T_{\mathrm{I}}+d_{\mathrm{II}} \Delta T_{\mathrm{II}}+d_{\mathrm{III}} \Delta T_{\mathrm{III}} .
$$

In most cases the damping factors can be chosen as unity, but in some problematic models the action of the three terms must be balanced by hand.

- Different convergence speeds from the three terms of Eq. (64) is in general the main problem of this method. As in converged Wolf-Rayet model atmospheres the temperature is usually monotonically decreasing with radius, it is often useful to restrict the corrections such that $T(r)$ remains monotonic during the whole iteration process.

- For similar reasons it is often useful to smooth the temperature corrections, e.g. by replacing the correction $\Delta T_{l}$ obtained at radius index $l$ by a mean including adjacent points, $\frac{1}{4} \Delta T_{l-1}+\frac{1}{2} \Delta T_{l}+\frac{1}{4} \Delta T_{l+1}$.

\section{Example results}

The temperature correction method described above has been successfully employed for calculating extended grids of model atmospheres for Wolf-Rayet stars. Our models (see



Fig. 2. A model which is not yet in Radiative Equilibrium (see text for model parameters). The upper panel shows the co-moving frame flux, expressed in terms of the radiation temperature, as function of the radius index. The outer boundary is at index 1 (left). The intended effective temperature of the model is given by the dashed horizontal line. The flux which the model should have in Radiative Equilibrium is somewhat lower in the outer parts (solid line). The actual flux of the model in that stage (circles) still deviates from that by much. The temperature corrections which our method applies in this situation are shown in the lower panel. The contributions from the three terms in Eq. (64) are shown separately: the local Radiative Equilibrium (thin solid line), the term driven by the radius-integrated flux error (dashed line), and the term from the flux error at the outer boundary (dashdotted line). The total temperature correction (thick solid line) is the sum of the three contributions, all of them damped here with a factor 0.7 (Eq. (76)).

Gräfener et al. 2002 for more details) usually account for hydrogen, helium and CNO with complex model atoms, the composition being typical either for $\mathrm{WN}$ or for $\mathrm{WC}$ stars. Iron line blanketing is treated in the superlevel approximation.

The model which will serve here for demonstrating the temperature correction method has been calculated for the enigmatic WN-type central star of the planetary nebula N 66 in the Large Magellanic Cloud (Hamann et al. 2003). The model parameters are: effective temperature $T_{*}=112.2 \mathrm{kK}$, luminosity $\log L / L_{\odot}=4.78$, mass-loss rate $\log \dot{M} /\left(M_{\odot} \mathrm{yr}^{-1}\right)=-5.386$ with clumping factor $D=4$, and terminal wind speed $v_{\infty}=$ $2200 \mathrm{~km} \mathrm{~s}^{-1}$. The chemical composition is (by mass fraction) 0.2 hydrogen, 0.003 nitrogen, $1 \times 10^{-4}$ carbon, $2 \times 10^{-4}$ iron group elements, and the rest helium. 


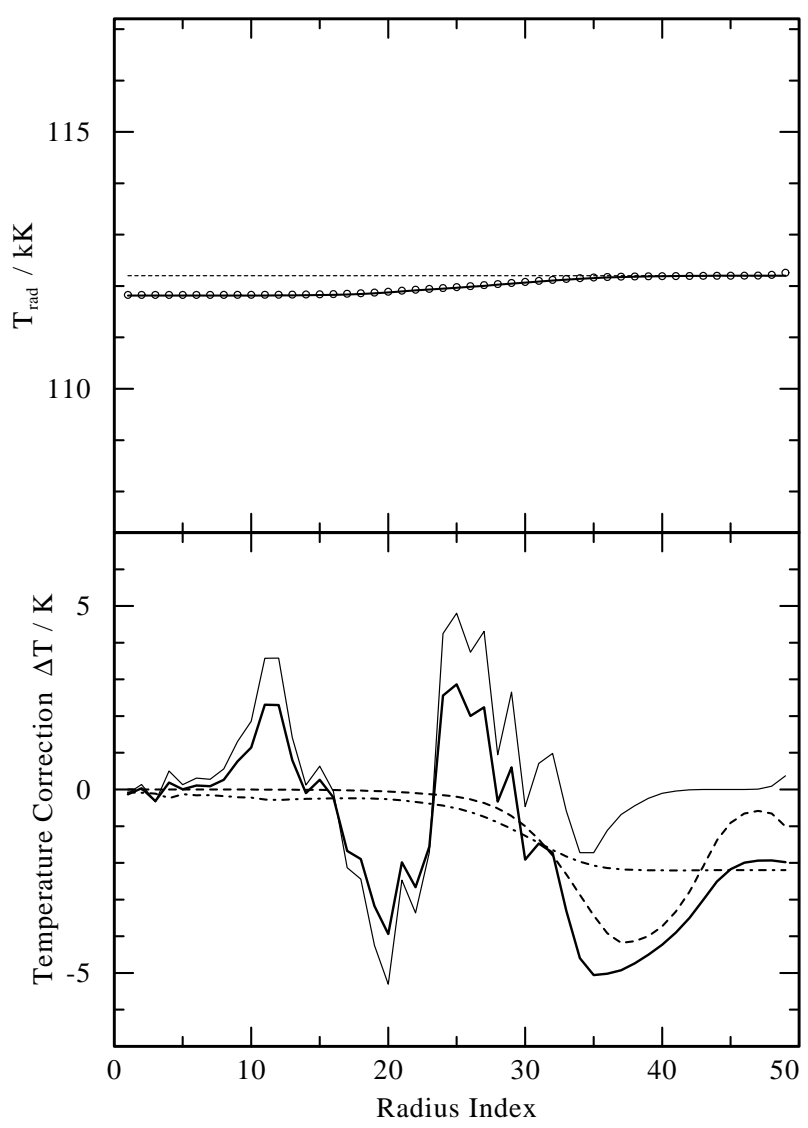

Fig. 3. Same as Fig. 2, but many iterations later. As seen in the upper panel, the model flux (circles) now perfectly fulfills Radiative Equilibrium (thick full line). The remaining temperature corrections (lower panel) applied by our method are now only a few Kelvin (note the expanded scale).

Figure 2 refers to an early stage of the iteration, when the model is still far from Radiative Equilibrium. The upper panel shows the co-moving frame flux, expressed in terms of the radiation temperature, as function of the radius index. The intended effective temperature of the model is given by the dashed horizontal line. The flux which the model should have in Radiative Equilibrium (solid line) is somewhat lower in the outer parts of the atmosphere, because the photon field looses energy by mechanical work (cf. Eq. (63)). The actual flux of the model in that stage (circles) differs by much from the correct values. The temperature corrections which our method applies in this situation are shown in the lower panel.

Figure 3 shows the same model, but many iterations later. As seen in the upper panel, the model flux now perfectly fulfills Radiative Equilibrium (thick full line). The remaining temperature corrections (lower panel) are very small.

In the outer part of the atmosphere, only the local energy balance yields significant temperature corrections. Here the acceleration of the iteration (ALO method, Sect.3.5) is essential. The convergence is checked by the following test. Figure 4 shows the temperature stratification for the same model as in the previous figures. The full line gives $T(r)$ of the converged model. Now this stratification is artificially

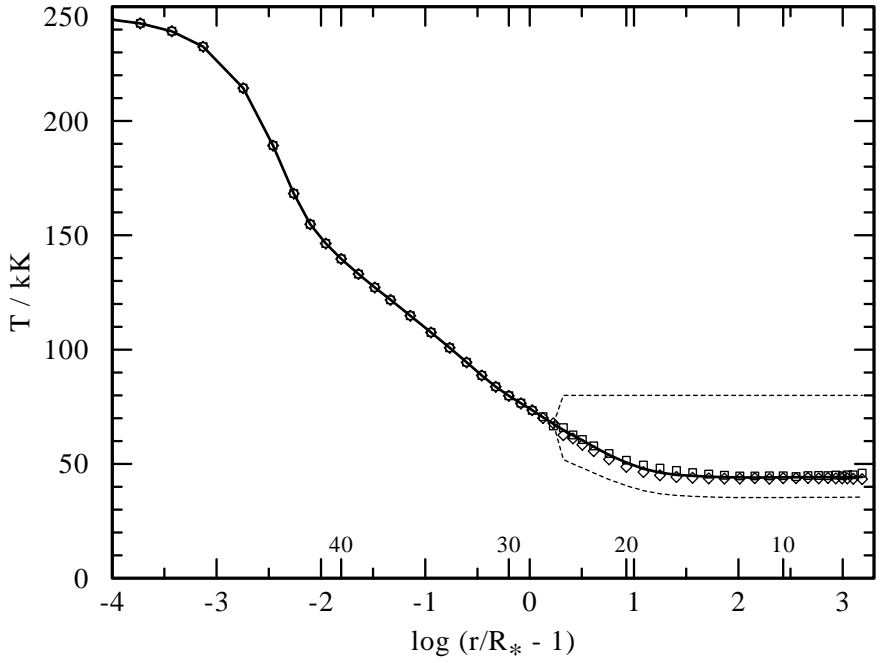

Fig. 4. Temperature stratification for the same model as in the previous figures, plotted versus radius $r$ in units of the stellar radius $R_{*}$. The radius point indices are also indicated along the abscissa axis. The full line gives the stratification of the converged model. For two convergence tests, this stratification was artificially modified in the outer part (dashed lines). After running further iterations, both of these manipulated models (squares and diamonds for the models (i) and (ii), respectively) recover the original, converged temperature stratification.

modified in the outer part, setting the temperature (i) to $80 \mathrm{kK}$ and (ii) to 0.8 times the converged values, respectively (dashed lines). After running further iterations, both of these models recover the converged temperature stratification (squares and diamonds for the models (i) and (ii), respectively), thus successfully demonstrating convergence.

In summary, we have presented and tested a temperature correction method for computing Radiative Equilibrium models of spherically expanding stellar atmospheres in non-LTE.

Acknowledgements. We thank A. Feldmeier for many discussions and careful reading of the manuscript.

\section{References}

Gräfener, G., Koesterke, L., \& Hamann, W.-R. 2002, A\&A, 387, 244

Hamann, W.-R. 1985, A\&A, 148, 364

Hamann, W.-R. 1986, A\&A, 160, 347

Hamann, W.-R. 2003, in Stellar Atmosphere Modelling, ed. I. Hubeny, D. Mihalas, \& K. Werner, ASP Conf. Ser., 288, 171

Hamann, W.-R., \& Wessolowski, U. 1990, A\&A, 227, 171

Hamann, W.-R., Peña, M., Gräfener, G., \& Ruiz, M. T. 2003, A\&A, 409, 969

Hauschildt, P. H. 1992, ApJ, 398, 224

Lucy, L. B. 1964, in Proceedings First Harvard-Smithsonian Conference on Stellar Atmospheres, Cambridge, Massachusetts, SAO Special Report, 167, 93

Mihalas, D. 1978, Stellar Atmospheres, 2nd ed. (San Francisco: Freeman)

Unsöld, A. 1951, Naturwiss, 38, 525

Unsöld, A. 1955, Physik der Sternatmosphären, 2nd ed. (New York, Heidelberg: Springer-Verlag Berlin) 